\title{
Dust charging and density conditions deduced from observations of PMWE modulated by artificial electron heating
}

\author{
O. Havnes, ${ }^{1}$ C. La Hoz, ${ }^{1}$ M. T. Rietveld, ${ }^{2}$ M. Kassa, ${ }^{1}$ G. Baroni, ${ }^{1}$ and A. Biebricher ${ }^{1}$ \\ Received 17 June 2011; revised 26 October 2011; accepted 27 October 2011; published 29 December 2011.
}

[1] We present an analysis of relatively strong Polar Mesospheric Winter Echoes (PMWE) under artificial electron heating that changes the PMWE intensity. A major purpose is to find reliable estimates of the relaxation time of the heater modified PMWE to their undisturbed state during the heater switch-off phase; the implications regarding charge/ discharge mechanisms; and to exploit the diagnostic potential of artificial electron heating. The relaxation time is between 60 to $70 \mathrm{~s}$ for the regions with strong PMWE layers and substantial electron heating. This short relaxation time, related to the variation of charges on the nanometer dust which most likely is present in PMWE, rules out ion attachment as the mechanism to bring the dust charges to their equilibrium state. Neutral winds, sweeping the heated electrons out of the radar beam, are unlikely to be the cause of the observed relaxation, since this requires winds of around $100 \mathrm{~m} \mathrm{~s}^{-1}$. The most probable cause is photo detachment by which negatively charged dust can lose excess electrons by photon absorption with energies less than the dust material's work function. By comparing the observed heating with heating model profiles, the electron density at $65 \mathrm{~km}$ height must have been of the order of $3 \times 10^{9} \mathrm{~m}^{-3}$. This agrees with PMWE occurring mainly during disturbed conditions with high electron densities. Our results also indicate that in the strongest PMWE layers, electron bite-outs exist consistent with the role of charged dust particles in the mechanism of PMWE and implying larger dust densities.

Citation: Havnes, O., C. La Hoz, M. T. Rietveld, M. Kassa, G. Baroni, and A. Biebricher (2011), Dust charging and density conditions deduced from observations of PMWE modulated by artificial electron heating, J. Geophys. Res., 116, D24203, doi:10.1029/2011JD016411.

\section{Introduction}

[2] Polar mesospheric summer echoes (PMSE) are enhanced radar echoes originating from the polar mesopause around heights between 80 and $90 \mathrm{~km}$ that occur only in summer when the temperature near the mesopause becomes low enough for ice particles to be formed [Rapp and Lübken, 2004; Chilson et al., 2000]. It has been established that the source of the electron irregularities that produce the enhanced scattering is controlled by submicroscopic nanometer-sized charged dust particles. There is compelling evidence that the particles are composed of water molecules that can condense only during the cold summer temperatures of the polar mesosphere and can be charged by electron thermal currents. These are the same particles (charged or not) that produce noctilucent clouds, although only the ones with sizes of a few tens of nanometers are visible. The more recently discovered enhanced radar echoes, Polar Mesospheric Winter Echoes (PMWE), have some seasonal dependence, possibly disappearing during the PMSE season, and occur rather below the mesopause over

\footnotetext{
${ }^{1}$ Institute of Physics and Technology, University of Tromsø, Tromsø, Norway.

${ }^{2}$ EISCAT Scientific Association, Ramfjordbotn, Norway.

Published in 2011 by the American Geophysical Union.
}

a wider altitude range than PMSE besides being considerable weaker and much less frequent [Zeller et al., 2006]. The understanding of PMWE is less developed than that of PMSE and there is debate in the literature whether the scattering mechanism is the same or similar to PMSE or different. The present investigation, grounded in previous experimental and theoretical developments, takes the point of view that it is similar, the charged dust particles being nanometer or subnanometer-sized meteoritic smoke. Besides the primary interest in understanding nature, these phenomena afford a means to investigate the dynamics of the regions where they occur (especially PMSE, which is more frequent) as they act as tracers (winds, waves and turbulence) and as a plasma laboratory to study dusty plasmas, as it is possible to modify these phenomena artificially in a controlled manner with an RF heater.

[3] Artificial electron heating [Rietveld et al., 1993] can be used to investigate mesospheric phenomena such as the polar mesospheric summer echoes (PMSE) [Chilson et al., 2000] by using a heater cycling with $10 \mathrm{sec}$ on and $10 \mathrm{sec}$ off, observed that the PMSE radar backscatter could be made to practically disappear when the heater was switched on and thereafter return to its original strength when the heater was switched off. By running the heater with a relatively short on time $(20 \mathrm{sec})$ and a long off time $(160 \mathrm{sec})$ a PMSE overshoot phenomena can be created. In this case, the PMSE backscatter will also be reduced in intensity as the heater is 
switched on, but as the heater is switched off it will nearly instantaneously overshoot by a factor up to 6-7 times that of the value before the heater was switched on [Havnes, 2004; Havnes et al., 2003; Kassa et al., 2005]. The variation of the backscatter intensity profile during a heater on/off overshoot cycle (OCC: Overshoot Characteristic Curve) contains information on the dust and plasma conditions and can be used to diagnose the dusty plasma [Havnes et al., 2003; Biebricher et al., 2006; Scales and Chen, 2008].

[4] PMWE occurs mostly outside the summer months at heights from $\sim 55$ to $80 \mathrm{~km}$ [Zeller et al., 2006] as opposed to the summer echoes (PMSE) that occur only in summer and exclusively near the mesopause in the height range 80 $90 \mathrm{~km}$. The mechanism of PMSE involves charged ice particles of size from few nanometers to several tens of nanometers (see Rapp and Lübken [2004] and Friedrich and Rapp [2009], and references therein, for comprehensive reviews on PMSE). The mechanism of PMWE apparently also involves solid particles but of a different nature. These are probably very small nanometer particles of meteoric origin [La Hoz and Havnes, 2008; Havnes and Kassa, 2009], since the mesospheric temperature outside the summer months is too high for ice particles to form. Besides the purely intrinsic scientific interest to understand how natural phenomena occurs (what is the mechanism that enhances the radar echoes?), interest in PMWE is motivated by its likely connection to meteoritic smoke of which not much is known [Rosinski and Snow, 1961; Hunten et al., 1980; Kalashnikova et al., 2000; Megner et al., 2006], although due to the increasing interest, new observations employing various techniques are appearing [Hervig et al., 2009; Robertson et al., 2009; Rapp et al., 2010]. Added to this is also the experimental advantage of artificially modifying PMWE by artificial electron heating [Kavanagh et al., 2006; Belova et al., 2008; La Hoz and Havnes, 2008; Kero et al., 2008; Havnes and Kassa, 2009]. Models of the effects of artificial electron heating on PMSE have been very valuable to validate theories of PMSE as well as their use as a powerful diagnostic tool; the same advantages exist for the investigation of the winter echoes. PMWE signals are much weaker than PMSE and the overshoot factor is also much weaker than that of PMSE. Typically, strong PMWE layers are a factor of $10^{4}$ weaker than strong PMSE at $53.5 \mathrm{Mhz}$ [Zeller et al., 2006]. Long observation series of relatively strong PMWE are needed to obtain reliable statistics on PMWE and the effect of dust.

[5] Kero et al. [2008] suggest that a change of the density of negative ions during a heater cycling could influence the PMWE due to a variation of electron density that results from their attachment to neutral particles to form negative ions. They model PMWE variations observed with the EISCAT VHF radar under a heater cycle of $6 \mathrm{sec}$ on and $6 \mathrm{sec}$ off by the decrease of electron density due to increased formation of negative ions when the electrons are heated. However, we find that this cannot be a dominant mechanism for the variation of the PMWE strength during heater cycling. PMWE, when acted upon by the heater, can be weakened by a factor as high as 10 or more on a timescale of less than a second [La Hoz and Havnes, 2008] (see also Figure 3 in section 2 ). The change in electron density by the formation of negative ions is a much slower process occurring over a time of 5 to 6 seconds and leads to a reduction of PMWE strength by a factor of only around two [Kero et al., 2008]. When the heating is switched off, the electron density returns to its original value on a similar timescale without overshoot. However, we cannot exclude that the mechanism suggested by Kero et al. [2008] can have some influence on the shape of the overshoot curve, possibly being part of the explanation of the delayed maximum seen in the overshoot $[\mathrm{La} \mathrm{Hoz}$ and Havnes, 2008], which also is apparent in Figure 3. Havnes et al. [2009] suggest that this delay could be caused by spatial diffusion effects on the smallest dust particles.

[6] In this paper we examine observations of PMWE at $56 \mathrm{MHz}$ affected by artificial electron heating that were stronger and lasted longer than previous observations. The paper of La Hoz and Havnes [2008] reported the first observations of PMWE with the MORRO radar and concluded that a weak overshoot was present following the turning off of the heater. The paper of Havnes and Kassa [2009] concentrated on theoretical and modeling grounds the possible mechanisms for the creation of a heater induced weak overshoot, especially the charging/discharging mechanisms that may produce an overshoot, and made a short, passing reference to the experimental results of the La Hoz and Havnes [2008] paper. The new data set with stronger PMWE signals used in the present investigation has permitted us to make a comprehensive comparison and test for the first time the hypothesis proposed in the 2009 paper on the dominant role of various charging/discharging mechanisms by obtaining for the first time reliable estimates of the relaxation time of the overshoot signals. The estimated relaxation times and their implication on charging/discharging mechanisms are presented in Section 2, while in Section 3 we investigate whether the heating effects as a function of height show signatures of electron bite-outs, which appears to be the case confirming the role of charged dust in the mechanism of PMWE and implying larger dust densities. A summary of the results and the conclusions are presented in Section 4.

\section{The Relaxation Time in PMWE Overshoot Experiments}

[7] We have analyzed the overshoot effects excited by the EISCAT RF heater in a relatively strong PMWE layer observed on the 12th February 2008 by the $56 \mathrm{MHz}$ MST MORRO radar at the EISCAT site near Tromsø, Norway $\left(69^{\circ} 35^{\prime} \mathrm{N}, 19^{\circ} 14^{\prime} \mathrm{E}\right)$ (http://tupac.phys.uit.no/ cesar/ MORROradarSite/MORROradar.html). The MORRO radar operated at full power (96 kW peak) and nearly full duty cycle $(8.5 \%$ of maximum $10 \%)$ employing complementary codes to yield an altitude resolution of $300 \mathrm{~m}$; while the time resolution was sub-second, however, the signal power estimates employed here were time integrated with 2 second resolution. The heater was operated with a vertical antenna beam and the transmitter configured to send $\mathrm{X}$-mode waves of frequency $5.423 \mathrm{MHz}$ delivering $890 \mathrm{~kW}$ of power. The antenna gain of $27.56 \mathrm{dBi}$ resulted in an effective radiated power of approximately $507 \mathrm{MW}$.

[8] Figure 1 shows a plot of the signal intensity (color scale) as a function of height (vertical axis) and profile number (horizontal axis). The first record starts at $08 \mathrm{~h} 23 \mathrm{~m}$ UT and coincides with the instant when the first heater cycle was switched on. The effect of the heater is clearly seen as the regularly spaced darker vertical bands coinciding with 


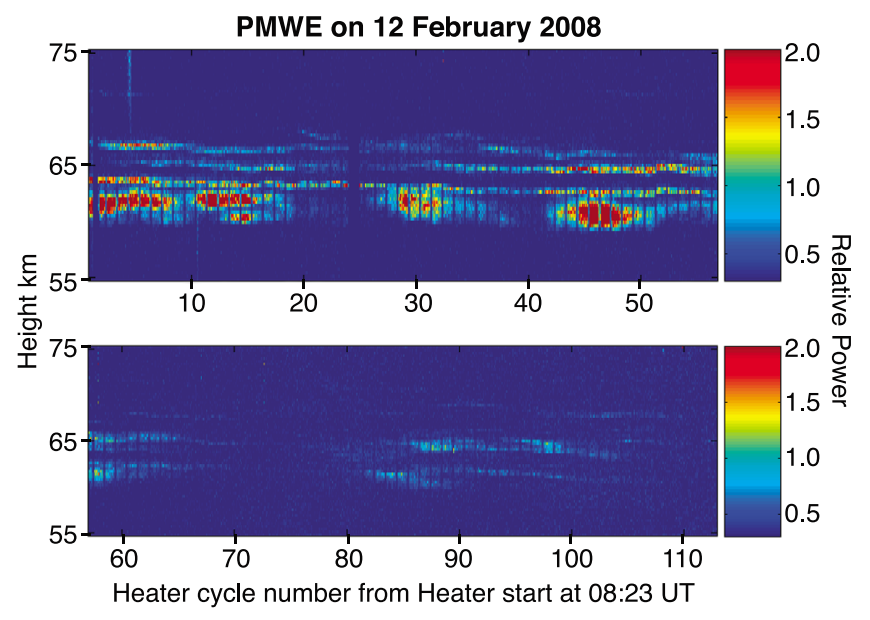

Figure 1. An overview of the observed PMWE on 12.02.2008 from the onset of the heater at time 0823 UT. The heater cycle is $2 \mathrm{~min}$. The PMWE which are analyzed are up to cycle 67.

the heater on periods when PMWE is weakened. The observations were inspected for strong outliers that could significantly affect our analysis resulting in one heater cycle (number 24) being discarded. The heater was run with repeated cycles of $20 \mathrm{sec}$ with the heater on and $100 \mathrm{sec}$ with the heater off, a total of 2 min per cycle. This choice was made at the time on preliminary analysis and later justified by the thorough analysis of the factors influencing the relaxation time of PMWE from Havnes and Kassa [2009], who argued that the most likely dominating relaxation mechanism could be photo-detachment, which appears to be confirmed by this experimental investigation. The judgement on reducing the cycle time with respect to previous experiments was also strongly influenced by a desire to increase the statistics as much as possible knowing that PMWE signals have low signal-to-noise-ratio, SNR: a shorter cycle time will produce a greater number of cycles for a given time interval.

[9] The overshoot effect is basically caused by dust particles acquiring additional negative charges during the time the heater is on. The charged dust will then exert an increased influence on the electron density distribution when the heater is switched off, compared to that before the heater was switched on. This will steepen electron density gradients and leads to an increased radar backscatter - an overshootwhen the heater is switched off [Havnes, 2004; Havnes et al., 2004; Kassa et al., 2005; Biebricher et al., 2006]. After the heater is switched off, the dust particles gradually lose their excess negative charges and their influence on the electron density distribution reverts to that of the undisturbed conditions, as does the PMWE signal strength. The relaxation time of PMWE intensity will give information on the dominant charging/discharging processes that lead to the reduction of the negative dust charges acquired during the heating phase such that the dust particles return to their undisturbed charge state. To investigate the relaxation time we have chosen an interval when PMWE was stronger, namely the first $\sim 2$ hours up to heater cycle 67 of Figure 1 . We see from Figure 1 that the strength of PMWE is largest in the lower layers. This is also apparent in Figure 2 (left) which shows the height distribution of the signal plus noise to noise ratio $(\mathrm{S}+\mathrm{N}) / \mathrm{N}$, where $\mathrm{S}$ is the signal power and $\mathrm{N}$ is the noise power. The noise power was estimated by averaging the power at altitude ranges below $(50-53 \mathrm{~km})$ and above $(87-90 \mathrm{~km})$ the PMWE where no signal was present. In our analysis we split the PMWE height region into 3 parts where the lowest region up to $62 \mathrm{~km}$ contains the strongest PMWE signals while the upper layer above $65 \mathrm{~km}$ contains the weakest layers. On Figure 2 (right) we show the distribution of the ratio by which the radar backscatter weakens when the heater is switched on, R1/R0, as a function of $(\mathrm{S}+\mathrm{N}) / \mathrm{N}$. Here R0 is the radar backscatter intensity corrected for noise just before the heater is switched on; while R1 is the backscatter, also corrected for noise, just after the heater has been switched on causing a weakening of PMWE, see Figure 3; in other words, R1/R0 is the factor by which PMWE is weakened nearly instantaneously just after the heater has been turned on and is an indirect measure of the extent to which the electrons have been heated which can be parameterized in the models, thereby its double importance. As we will discuss in Section 2, strong heating results in deep weakening of the signal (a small value for R1/R0), sometimes down into the noise level, R1/R0 being zero in this case. If there were no heating effects, R1/R0 would be equal to 1 . It is apparent that the heating effect is largest in the lowest height region with the strongest PMWE and is weakest in the upper height region where PMWE is also weak. It is a relevant question why the weakening of the signals after heater turn on varies with height. The answer lies most likely in how the electron heating due to the heater wave varies as a function of height. The variation of heating with height is controlled by the electron density height profile [Belova et al., 1995; Kassa et al., 2005; Routledge et al., 2011]. The heater wave energy is absorbed by electrons and lost mainly in collisions with neutrals. At quiet conditions, with low electron density in the lower regions $(60-70 \mathrm{~km})$, little of the heater wave energy will be absorbed there and one will see a substantial heating also above this height. Since the maximum heating of the electrons depends on the loss rate through collisions with neutrals, the heating can be much higher in the upper parts of the mesosphere than in the lower parts. For disturbed conditions with high electron densities in the lower parts, most of the wave energy is absorbed low down and little is left to heat the upper layers. We see this effect clearly in Figure 5 (in section 3) where an increased electron density leads to a decrease of the heating in the upper parts.

[10] The overshoot in a very weak signal is probably undetectable due to the noise with the short integration times that have been used. We expect that the most reliable results on the relaxation time will be for the lower height region where PMWE is strongest and the heating effect also strongest. In the uppermost height region with the weakest PMWE the overshoot will be weak with an uncertain relaxation phase and a corresponding unreliability of the relaxation times that could be found. From Figure 2 we see that the values of $\mathrm{R} 1 / \mathrm{R} 0$ for the lowest height region are all well contained below a value of 0.5 corresponding approximately to a doubling of the electron temperature caused by heater turn on (see equation (4)). However, for the upper height region, the $\mathrm{R} 1 / \mathrm{R} 0$ values are fairly evenly distributed between 0 and 1 showing that the heating effects are small 

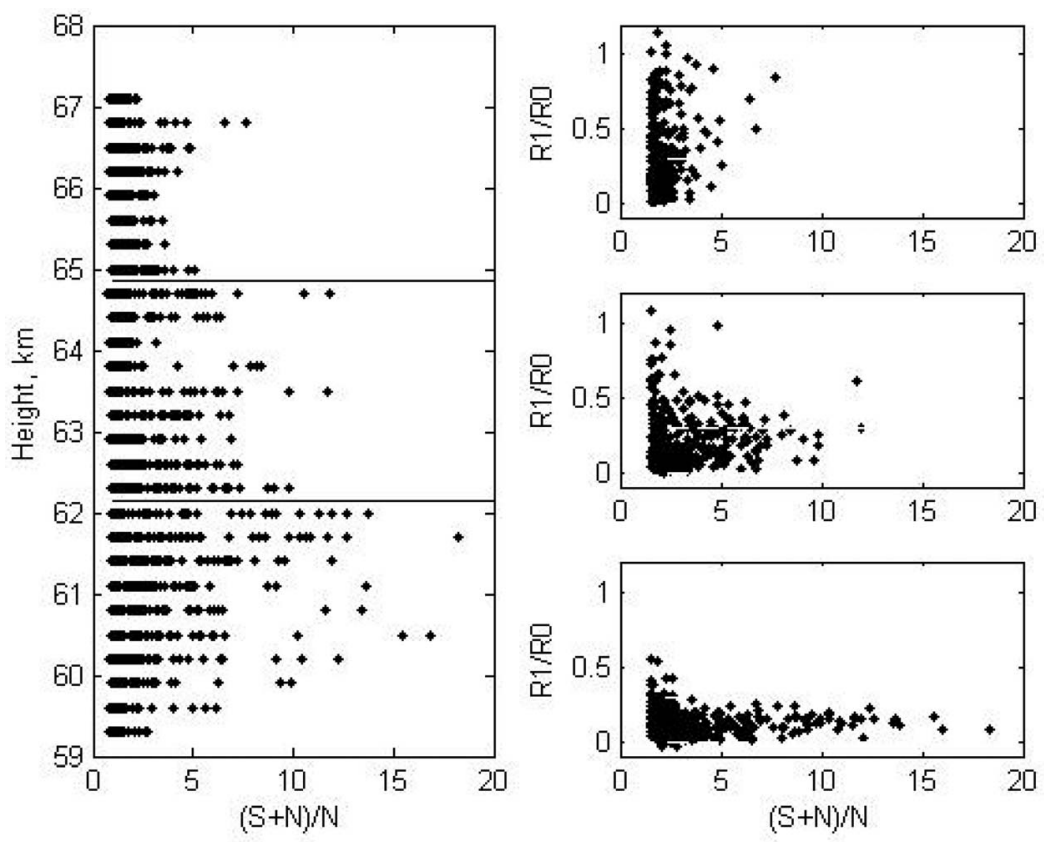

Figure 2. (left) The height distribution of the $(\mathrm{S}+\mathrm{N}) / \mathrm{N}$, the ratio of (signal + noise) to noise. We have considered 3 different height regions which are divided by the horizontal black lines. (right) The distribution of signal weakening ratio, R1/R0, for each of the three height regions. Low values of R1/R0 represent a signature of large electron heating. See the text for details.

and that additive random noise and the intrinsic variability of the signals are probably major effects in creating the observed distribution.

[11] An estimate of the relaxation time $t_{R}$ was performed by averaging the first 68 OCC curves located in the height interval between 58.1 and $63.2 \mathrm{~km}$ corresponding to 18 radar height ranges each of $300 \mathrm{~m}$. The choice of the altitude interval was made in order to include the PMWE region that contains the main heating effects. As discussed previously, the heating intensity (the enhancement of the electron

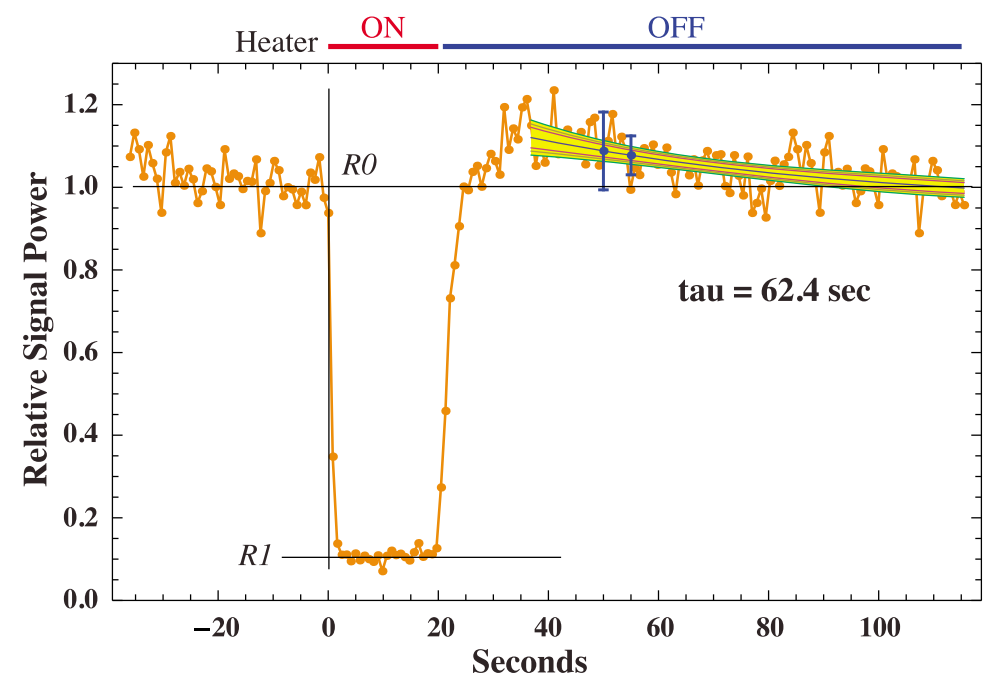

Figure 3. The average overshoot curves of the first 68 cycles and for the lower height region between 58.1 and $63.2 \mathrm{~km}$. Above this height interval the heating effects were small. The average includes OCCs with moderate to strong heating, meaning $\mathrm{R} 1 / \mathrm{R} 0<0.3$ and with a threshold of $(\mathrm{S}+\mathrm{N}) / \mathrm{N}>2$ (see main text). The number of OCC periods employed in the average is 381 . The relaxation time $t_{R}=62.4 \mathrm{sec}$ was calculated by fitting a decaying exponential function with origin $30 \mathrm{~s}$ after the heater was switched on. The fitted exponential is shown in red. Two colored curves embrace the $95-99 \%$ confidence interval of the fitting. The two error bars shown are the $\pm 1 \sigma$ and $\pm 2 \sigma$ variances obtained from the fitting residuals. See the text for additional details. 
temperature) above this region was manifestly small and the unmodified PMWE weaker with the result that the overshoot effect is absent or, more likely, dominated or masked by random fluctuations of the signal and the noise. The average includes OCCs with moderate to strong heating, meaning $\mathrm{R} 1 / \mathrm{R} 0<0.3$ and with a threshold of the ratio between signal plus noise to noise $(\mathrm{S}+\mathrm{N}) / \mathrm{N}>2$ (or equivalently $\mathrm{S} / \mathrm{N}>1$ ). The number of OCC periods employed in the average is 381 . Each individual OCC was normalized to 1 just before the heater was switched on (R0) and thereafter averaged. The result of the averaging is illustrated in Figure 3. A decaying exponential with origin 30 seconds after the heater was switched on was fitted to the average OCC, also shown in Figure 3, which resulted in a relaxation time of $t_{R}=62.4$ seconds. Exponential decay is the most frequent form of relaxation processes; it appears as a natural for our case and is confirmed by the quality of the statistics involved in the fitting. The confidence level of the estimated relaxation time is good as inferred from the statistical goodness of fitting as expressed by the confidence interval curves at the 95-99\% levels shown in Figure 3 as the colored region embracing the fitted curve. The two error bars in Figure 3 represent the $\pm 1 \sigma$ and $\pm 2 \sigma$ standard errors obtained from the fitting residuals. The standard $(95 \%)$ confidence interval of the fitted time constant is $[44.2,80.6] \mathrm{s}$, meaning that only one out of 20 measurements of the time constant will be outside this interval. Alternatively, the $\pm 1 \sigma$ error bar of the estimated time constant is $\pm 9.2 \mathrm{~s}$. The alternative discharge mechanism via ion attachment has relaxation times about an order of magnitude larger, as we proceed to show in what follows.

[12] The question is then which discharge/charge mechanisms can lead to the observed relaxation times of the order of 60 seconds? For the PMSE summer radar clouds the relaxation time is apparently also around a minute [e.g., Kassa et al., 2005] and can be explained by the fact that the increased negative charges on the dust particles acquired during the heater on phase relax to the undisturbed charge values by ion attachment [Havnes et al., 2004]. However, while PMSE contains comparatively large particles from $\sim 10 \mathrm{~nm}$ to $\sim 100 \mathrm{~nm}$ which are often visually observable [von Cossart et al., 1999], PMWE most probably contains very small, non-visual dust particles of radius from $\sim 3-4 \mathrm{~nm}$ and smaller [Havnes and Kassa, 2009]. Since the collision frequency between plasma particles and charged dust decreases rapidly with dust size and dust charge, ion attachment cannot explain the relaxation time of PMWE. The collision frequency for a case with attraction force between the plasma particle and charged dust particle is given by [Weingartner and Draine, 2001; Shukla and Mamun, 2002]

$$
v_{C}=n_{i}\left(8 k T_{i} / \pi m_{i}\right)^{1 / 2} \pi r_{d}^{2}\left(1-Z_{i} e U / k T_{i}\right) F_{p}
$$

where the dust surface potential is

$$
U=Z_{d} e / 4 \pi \epsilon_{0} r_{d}
$$

The parameter $F_{p}$ is a correction for the short-range focusing due to polarization forces that will increase the effective cross section for plasma particle capture by dust. From the expressions given by Draine and Sutin [1987], we find that
$F_{p} \sim 1.7$ for PMSE conditions and dust radius $r_{d}=20 \mathrm{~nm}$; and $F_{p} \sim 2$ for PMWE conditions with $r_{d}=3 \mathrm{~nm}$. In equations(1) and (2) $n_{i}, m_{i}, T_{i}$, and $Z_{d}$ are the ion density, mass, temperature and dust charge number respectively. For the ion charge number we use $Z_{i}=1$. The Boltzmann constant is $k$, the elementary charge is $e$, while the permittivity of free space is $\epsilon_{0}$. We use the same ion density $n_{i}=3 \times 10^{9} \mathrm{~m}^{-3}$ and mass $m_{i}=30 m_{H}$ for both PMSE and PMWE. The high plasma density for PMWE, which is much lower in height than PMSE, requires disturbed magnetospheric conditions with energetic particle precipitation [Zeller et al., 2006]. For the other parameters $\left(T_{i}, r_{d}, Z_{d}\right)$ we use $(150 \mathrm{~K}, 20 \mathrm{~nm},-2)$ for PMSE conditions and $(220 \mathrm{~K}, 3 \mathrm{~nm},-1)$ for PMWE. This gives a collision time, or attachment time for the ions onto dust of $t_{A}=40 \mathrm{sec}$ for PMSE conditions and $t_{A}=570 \mathrm{sec}$ for the PMWE conditions. Larger ion masses of $m_{i}=50 m_{H}$ will increase the attachment times which becomes 50 and $740 \mathrm{sec}$, respectively. The attachment time is inversely proportional to the ion density and changes in this parameter will therefore influence strongly its value. Since the attachment time will be close to the relaxation time, it seems clear that ion attachment can explain the PMSE relaxation times but that it cannot explain the relaxation of PMWE, if this is caused by a reduction of the negative charges of dust particles with sizes that we have used. For this to be the case, the ion density would have to be increased by a factor of 10 , to a value $\sim 3 \times 10^{10} \mathrm{~m}^{-3}$, which we find to be unrealistic. This value is several times larger than the ion densities measured during a very strong proton event on January 18, 2005 [Brattli et al., 2006] where the plasma densities at PMWE heights were exceptionally large [Lübken et al., 2006]. We will consider two other mechanisms by which the PMWE signal can relax to the undisturbed PMWE value: (I) The effect of neutral winds at the PMWE heights, and (II) the effect of photo-detachment [e.g., Weingartner and Draine, 2001; Havnes and Kassa, 2009; Rapp, 2009].

[13] The neutral gas wind will lead to transport of the plasma volume contained within the heater and radar beam. The plasma, containing dust particles that have been additionally charged during the heater on phase, will be transported outside the volumes illuminated by the antenna beams by the wind and will be replaced during the relaxation phase when the heater is off by unheated plasma. The MORRO radar beam and the heater beam full widths at halfpower at $65 \mathrm{~km}$ height are 7.6 and $8.6 \mathrm{~km}$ respectively. We have calculated the decrease of the PMWE signal due to neutral wind transport, by assuming circular radar and heating beams with diameters given above and show the results in Figure 4 . We see that winds of velocities of $100 \mathrm{~m} \mathrm{~s}^{-1}$ or more are required to cause relaxation times as observed for PMWE. This is very much above the observed mean zonal winds of 5 to $10 \mathrm{~m} \mathrm{~s}^{-1}$ and mean meridional winds of 0 to $-2 \mathrm{~m} \mathrm{~s}^{-1}$ which are observed between heights 70 to $\sim 90 \mathrm{~km}$ in February for latitudes around $70^{\circ} \mathrm{N}$ [Portnyagin et al., 2004]. Although the observations we have used to determine the relaxation time are located somewhat lower than the heights for the cited zonal and meridional winds, we find it very unlikely that neutral winds close to $100 \mathrm{~m} \mathrm{~s}^{-1}$ were present during the PMWE event we observed. We therefore feel safe in concluding that winds 


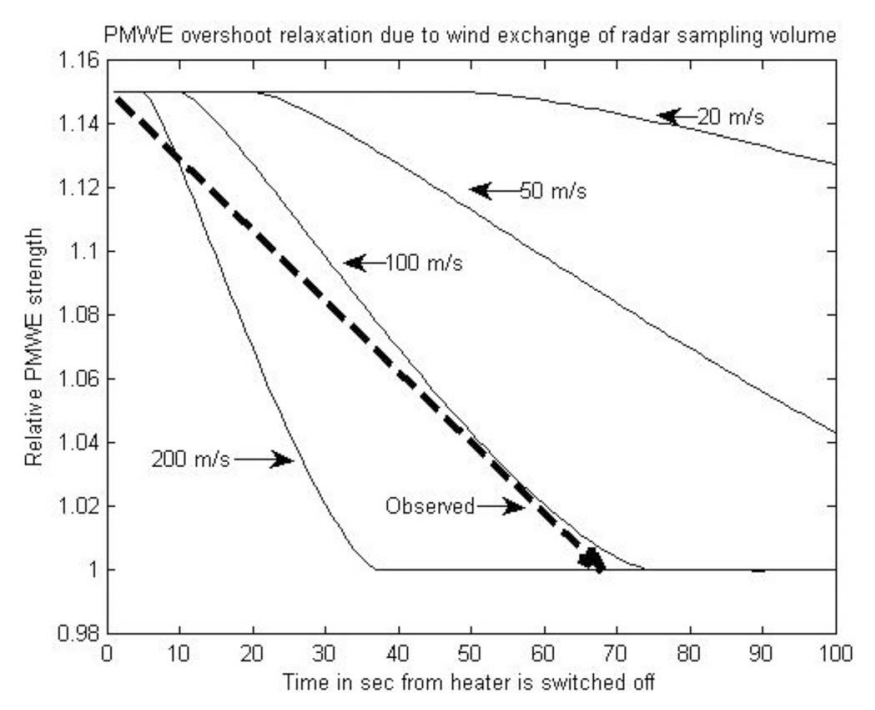

Figure 4. The decrease in PMWE signal as a function of time, for various wind velocities given here, assuming that this is the only effect which affects the observed PMWE signal by removing heated gas within the radar beam and replacing it with unheated gas. The broken line shows the observed relaxation from maximum signal back to an undisturbed signal in slightly less than $70 \mathrm{~s}$.

will rarely be major factors in causing the relaxation of PMWE overshoot signals.

[14] A more likely candidate that may explain the observed relaxation time is the photo-detachment effect, which is related to the photo-ionization effect and which occurs for negatively charged dust particles. Excess electrons can be ejected by a photon of energy considerably below the photoelectric work function of the dust bulk material [e.g., Weingartner and Draine, 2001]. During sunlit condition at the heights $60-65 \mathrm{~km}$ of these observations we may expect that energy radiation below $6.5 \mathrm{eV}$ (wavelength of $192 \mathrm{~nm}$ ) is present [Lean, 1987] and available for photodetachment. The threshold energy of this process with $Z=-1$ can be expressed as [Weingartner and Draine, 2001]

$$
(h v)_{e V}=W_{e V}-E_{b g}-2.2 / r_{n m}
$$

The band gap energy $E_{b g}=0$ for metals and semimetals, and of the order of $5 \mathrm{eV}$ for silicates. Since the work function $W_{e V}$ is likely to be $4-5 \mathrm{eV}$ for metals [Weingartner and Draine, 2001] and about $8 \mathrm{eV}$ for silicates we may have, if these numbers are representative, that the photon threshold energy for photo-detachment can be about 2.3 and $3.8 \mathrm{eV}$ for metal or semimetal grains and 0.8 and $2.3 \mathrm{eV}$ for silicates for grains sizes of 1 and $3 \mathrm{~nm}$ respectively.

[15] Havnes and Kassa [2009] and Rapp [2009] found that it is likely that photo-detachment is a dominant charging effect for nanometer particles in the mesosphere. Such small particles, expected to be produced when meteoric ablated material condenses into "smoke" particles [Rosinski and Snow, 1961; Hunten et al., 1980; Kalashnikova et al., 2000; Megner et al., 2006; Rapp et al., 2010], should mainly be electrically neutral during sunlit conditions. If the relaxation time we find is caused by the photo-detachment effect, we expect photo-detachment rates of $J_{\text {phd }} \sim 1 / t_{R} \sim 0.016$ electrons $\times$ particle $^{-1} \times$ second $^{-1}$ for 3-4 nm dust particles if they are responsible for the relaxation of the PMWE overshoot effect shown in Figure 3. If dust of $1 \mathrm{~nm}$ or less is also present, as predicted by modeling [Megner et al., 2006], these should have considerably larger photo-detachment rates [Weingartner and Draine, 2001; Havnes and Kassa, 2009]. With such values for the photo-detachment, the smallest and probably most numerous PMWE particles around $1 \mathrm{~nm}$ and less will stay mainly neutral at sunlight conditions [Havnes and Kassa, 2009] but become major sinks for electrons during night conditions. Even in cases when there is auroral particle precipitation at unusually high energy fluxes, the dust, in combination with negative ion production, should lead to significant electron density depletions during night conditions, which in turn results in a strongly reduced probability of observable PMWE under these conditions.

\section{The Heating Effect on PMWE}

[16] The immediate heating effect on PMWE (and PMSE) as the heater is switched on is given by the fraction by which the signal drops relative to the value just before the heater is switched on, that is, the ratio $R 1 / R 0$ introduced in Section 1 [Kassa et al., 2005]. Based on the assumption that dust irregularity structures control the electron density spatial structure, as is the case for PMSE, one can find expressions linking the ratio $R 1 / R 0$ to the electron temperature heating factor $T_{e, h o t} / T_{i}$ [Havnes et al., 2004; Kassa et al., 2005].

$$
\frac{T_{e, h o t}}{T_{i}}=\left(1+\frac{n_{i 0}}{n_{e 0}}-\sqrt{\frac{R 1}{R 0}}\right) /\left(\frac{n_{i 0}}{n_{e 0}} \sqrt{\frac{R 1}{R 0}}\right)
$$

Here the average ambient electron and ion density around the dust clumps are $n_{e 0}$ and $n_{i 0}$ respectively. $T_{e, h o t}$ is the heated electron temperature and $T_{N}$ the neutral gas temperature which is equal to the ion temperature $T_{i}$ and the unheated electron temperature. If PMWE (and PMSE) has low density of dust compared to the plasma, the electron density is little affected by dust absorption and the ratio $n_{i 0} / n_{e 0} \sim 1$. Equation (4) then reduces to

$$
\frac{T_{e, h o t}}{T_{i}}=\left(2-\sqrt{\frac{R 1}{R 0}}\right) /\left(\sqrt{\frac{R 1}{R 0}}\right)
$$

For cases with high dust density, an electron bite-out can be created in which the ion to electron density ratio can be considerably higher than 1 . In this case, equation (4) must be used which will give a lower heating factor than equation (5). For $n_{i 0} / n_{e 0} \gg 1$ it is lower by a factor $\sim 2$. In Figure 5 we show the result of finding the average heating effects as function of height for cycles 1 to 67 (see Figure 2), where we include all heights and $(\mathrm{S}+\mathrm{N}) / \mathrm{N}>1.5$. In order to obtain some information on possible variations of heating with time we have split the time interval into two parts. The red horizontal lines are from cycles 1-34 and the blue ones for cycles 35-67, each part containing observations covering $66 \mathrm{~min}$. In Figure 5 (left) the heating has been calculated by equation (5) for all cases. The right-hand side of the blue and red lines correspond to the values for the heating that is obtained directly by taking the average of the ratios $R 1 / R 0$ in height bins of $900 \mathrm{~m}$. The values are corrected for the average background 

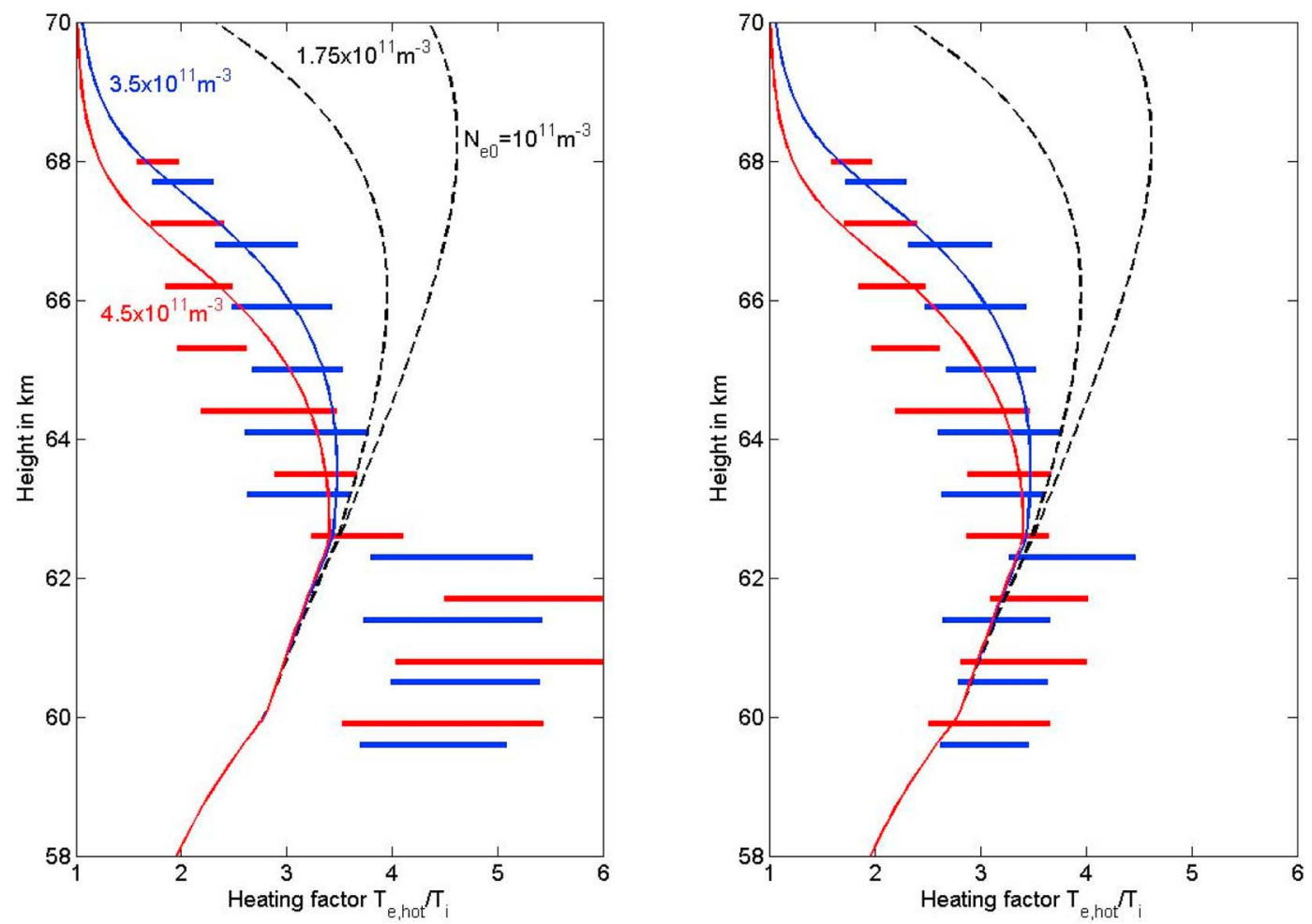

Figure 5. (left) The observed heating effects for the first half of the observing period as red horizontal lines and the last half as blue horizontal lines, calculated by equation (5). The length of the lines is associated with uncertainties in the temperature determinations. The red and blue curves are heating model fits to the observations of the red and blue cases. The required electron density at $90 \mathrm{~km}$, assuming an exponential decrease of density with decreasing height with scale height $5 \mathrm{~km}$, is also given for the respective cases. The broken lines are cases included to show the substantial change in the modeled heating curves for moderate changes in the electron density profiles. (right) The same results as in Figure 5 (left) with the exceptions that the heating from the observed average of R1/R0 now is calculated by equation (4) for heights below $62.5 \mathrm{~km}$, assuming an electron bite-out with an ion to electron density ratio of 5 . For details, see the text.

noise level, that is, we have employed the measured values of power with the noise subtracted. The value of R1 can be close to 0 since PMWE can be nearly suppressed during the heater on period when it is weak to start with. When this happens, an increase of the background by its uncertainty will often lead to values of R1 very close to 0 or even negative. In this situation, the $+1-\sigma$ confidence curve of the heating factor may diverge, which is unphysical. Therefore, we have chosen to include only the $-1-\sigma$ confidence level in Figure 5. These are the horizontal bars which start at the value of the heating factor on the right end of the line and drawn toward the left to cover the $-1-\sigma$ confidence level. By symmetry, one could argue that the $+1-\sigma$ side should not be much larger than the $-1-\sigma$ side. We see that the coloured lines together describe a curve that in Figure 5 (left) shows a peak in the lower and strong PMWE layers. Such a peak cannot be reproduced by theoretical heating curves [Kassa et al., 2005] and this suggests that $n_{i 0} / n_{e 0}>1$ in this region. If we assume the existence of an electron bite-out in the lower layers with $n_{i 0} / n_{e 0} \sim 5$ and use equation (4) to calculate the heating factor, we can get a very good fit of the theoretical heating to the observations throughout the PMWE layers as we show in Figure 5 (right). Such strong electron bite-outs in the region below $62 \mathrm{~km}$ do require dust densities that are higher than the electron densities, in our case densities well above $10^{9} \mathrm{~m}^{-3}$. We are unable to say if such dust densities are present in the lower layers of PMWE since we know of no direct measurements of nanometer-size particles during PMWE conditions. We find it intriguing that our observations apparently predict that high density layers can be present, possibly preferentially in the lower parts of PMWE. The densities given in Figure 5 (left) are the adopted electron densities at $90 \mathrm{~km}$ height for the various calculated heating curves. The electron density down into the atmosphere is assumed to decrease exponentially with a scale length $5 \mathrm{~km}$ and the heating variations with height are calculated with the model of Kassa et al. [2005]. The corresponding electron densities at $65 \mathrm{~km}$ are, from left to right, theoretical heating curves with values $(3,2.6,1.2,0.7) \times$ $10^{9} \mathrm{~m}^{-3}$. Apparently, the best correspondence between the theoretical and observed heating are for electron densities at $65 \mathrm{~km}$ height around $3 \times 10^{9} \mathrm{~m}^{-3}$ which is in good accordance with the densities found by Kavanagh et al. [2006] and Belova et al. [2008] from EISCAT VHF observations of PMWE. The peak of the theoretical heating curves are at lower altitudes when compared to other computations [e.g., Belova et al., 2001; Kassa et al., 2005; Routledge et al., 2011]. The explanation lies in the higher electron density in the 


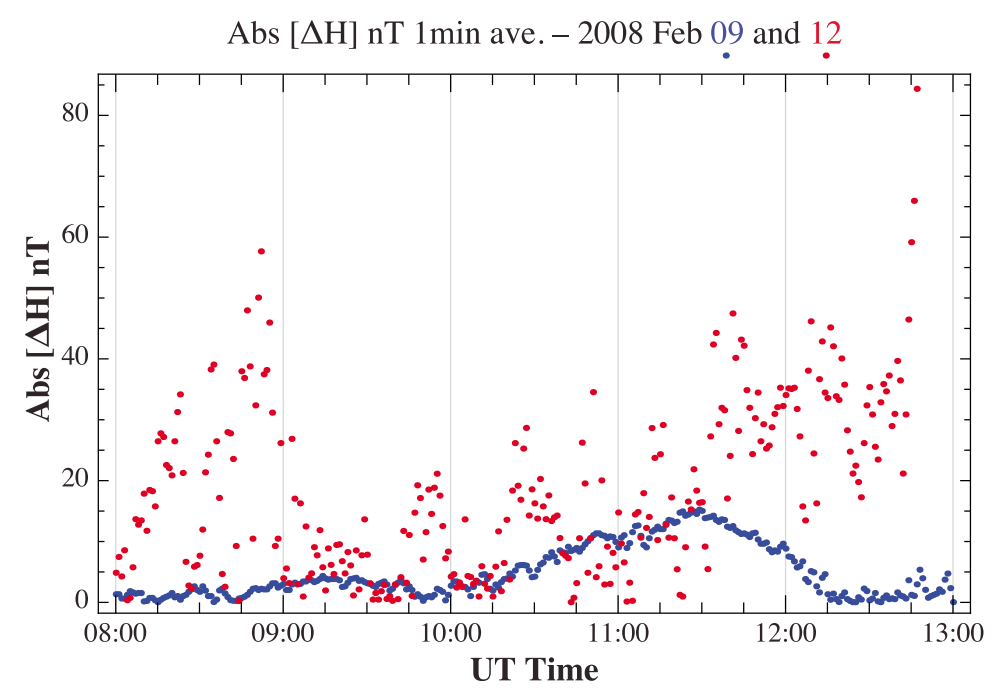

Figure 6. Tromsø Geomagnetic index, which is the horizontal component of the geomagnetic field in nanotesla, for the interval of time covering the measurements of PMWE reported here on the 12th of February 2008, in red dots. For comparison is also shown the index of the 9th of February, which was a quiet day. Note that the geomagnetic activity peaks shortly before 09:00 UT coinciding with the time where the more intense PMWE signals were measured. The K indices were 1 on the 9 th and 4 on the 12th of February.

mesosphere under PMWE conditions (at lower heights) which leads to increased heating energy being absorbed at lower altitudes which in turn lowers the height of maximum heating and produces a reduction of general heating.

[17] The results of Figure 5 also indicate that there has been a moderate decrease in the electron density from the first half of the observation period to the second half, since we see an increased heating in the upper part of the PMWE layer. This will result if there is a reduction in the precipitation during the two hours of observations that we have analyzed. This appears to be the case, since ionograms measured at the EISCAT site (not shown) and magnetometer observations from Tromsø Geophysical Observatory show higher activity in the first half of the observing period from 0823 UT to 0931 UT than in the second half from 0933 UT to $1041 \mathrm{UT}$ as can be seen in Figure 6 where the Troms $\varnothing$ index is plotted covering the period of these observations in red dots peaking shortly before 09:00 UT. It can be seen that there was moderate geomagnetic activity throughout the period corresponding to local $\mathrm{K}$ index of 4 . For comparison also shown is the index of the 9th of February (blue dots) that was very quiet with a $\mathrm{K}$ index equal to 1 .

\section{Conclusions}

[18] We have analyzed the results from an observation set where a fairly strong PMWE was modulated by artificial electron heating with the EISCAT Heating Facility [Rietveld et al., 1993] in an overshoot cycling [Havnes et al., 2004] of $20 \mathrm{sec}$ with heating followed by $100 \mathrm{sec}$ without heating. One of the aims of the analysis was to find reliable estimates for the relaxation time in the overshoot and identify the most likely mechanism controlling its relaxation. Although the observations are much influenced by noise and signal variations, the present cases show that the relaxation times in the low and strong layers most likely are in the range 60-
$70 \mathrm{sec}$. The higher and weaker PMWE layers could not give reliable results due to the weak signals and smaller heating effects. In contrast to what is the case for PMSE, which seems to have relaxation times of the same order as that for PMWE, we find that the PMWE overshoot relaxation cannot be caused by negative dust charge reductions caused by ion attachment, because of the postulated small size of the PMWE dust particles [La Hoz and Havnes, 2008; Havnes and Kassa, 2009; Belova et al., 2008]. We also found that wind transport of heated gas out of the radar beam requires exceptionally high neutral wind velocities of $\sim 100 \mathrm{~m} \mathrm{~s}^{-1}$, about an order of magnitude above the observed mean velocities [Portnyagin et al., 2004]. It appears that the most likely candidate to cause the observed relaxation rates is photo-detachment, an effect that recently has been suggested to be important for the small nano-meter sized meteoric dust particles in the mesosphere [Havnes and Kassa, 2009; Rapp, 2009]. Our findings lend support to these investigations and it seems clear that the charge state of such small particles must be different from what has earlier been assumed. With photo-detachment as a dominant charging effect, the smallest smoke particles should probably be mainly neutral at sunlit conditions while the larger ones may still be negatively charged as shown by Havnes and Kassa [2009]. During the night, however, without photo-detachment, also the smaller smoke particles can be negatively charged and will therefore represent a strong sink for electrons. This effect, together with the formation of negative ions [Kero et al., 2008] may affect the diurnal occurrence probability of PMWE and possibly explain why PMWE is nearly exclusively observed in daytime [Zeller et al., 2006].

[19] We have also shown that the heating effects on PMWE apparently are well described by existing heating models [Belova et al., 1995; Kero et al., 2000; Kassa et al., 2005]. A comparison between the heating effects found from observations and a heating model [Kassa et al., 2005] shows 
that the electron density must have been much higher than normal, which seems to be a requirement for PMWE to occur [Zeller et al., 2006]. We further showed that it is likely that the lower stronger PMWE layers produced an electron bite-out, corroborating the role of charged dust in the mechanism of PMWE and indicating that the dust particle density must have been high. We also found that changes with time of the observed heating profiles, as a function of height, can probably also be determined by changes in the electron density during the observation period, likely due to changes in the particle precipitation.

[20] Acknowledgments. This research was supported by grants from the Research Council of Norway. EISCAT is an international association supported by China (CRIRP), Finland (SA), Germany (DFG), Japan (STEL and NIPR), Norway (NFR), Sweden (VR) and United Kingdom (STFC).

\section{References}

Belova, E. G., A. B. Pashin, and W. B. Lyatsky (1995), Passage of powerful $\mathrm{HF}$ radio wave through the ionosphere as a function of initial electron density profiles, J. Atmos. Terr. Phys., 57, 265-272.

Belova, E., P. Chilson, M. Rapp, and S. Kirkwood (2001), Electron temperature dependence of PMSE power: Experimental and modeling results, Adv. Space Res., 28(7), 1077-1082.

Belova, E., M. Smirnova, M. T. Rietveld, B. Isham, S. Kirkwood, and T. Sergienko (2008), First observation of the overshoot effect for polar mesospheric winter echoes during radiowave electron temperature modulation, Geophys. Res. Lett., 35, L03110, doi:10.1029/2007GL032457.

Biebricher, A., O. Havnes, T. W. Hartquist, and C. La Hoz (2006), On the influence of plasma absorption by dust on the PMSE overshoot effect, Adv. Space Res., 38(11), 2541-2550.

Brattli, A., T. A. Blix, Ö. Lie-Svendsen, U.-P. Hoppe, F. J. Lübken, M. Rapp, W. Singer, and M. Friedrich (2006), Rocket measurements of positive ions during polar mesospheric winter echoes. Atmos. Chem. Phys., 6, 5515-5524.

Chilson, P. B., E. Belova, M. T. Rietveld, S. Kirkwood, and U.-P. Hoppe (2000), First artificially induced modulation of PMSE using the EISCAT heating facility, Geophys. Res. Lett., 27, 3801-3804.

Draine, B. T., and B. Sutin (1987), Collisional charging of interstellar grains, Astrophys. J., 320, 803-817.

Friedrich, M., and M. Rapp (2009), News from the lower ionosphere: A review of recent developments, Surv. Geophys., 30, 525-559, doi:10.1007/ s10712-009-9074-2.

Havnes, O. (2004) Polar mesospheric summer echoes (PMSE) overshoot effect due to cycling of artificial electron heating, J. Geophys. Res., 109, A02309, doi:10.1029/2003JA010159.

Havnes, O., and M. Kassa (2009), On the sizes and observable effects of dust particles in polar mesospheric winter echoes, J. Geophys. Res., 114, D09209, doi:10.1029/2008JD011276.

Havnes, O., C. La Hoz, L. I. Næsheim, and M. T. Rietveld (2003), First observations of the PMSE overshoot effect and its use for investigating the conditions in the summer mesosphere, Geophys. Res. Lett., 30(23), 2229, doi:10.1029/2003GL018429.

Havnes, O., C. La Hoz, A. Biebricher, M. Kassa, L. I. Næsheim, and T. Zivkovic (2004), Investigations of the mesospheric PMSE conditions by use of the new overshoot effect, Phys. Scr., 107, 70-78.

Havnes, O., C. La Hoz, M. T. Rietveld, M. Kassa, G. Baroni, and A. Biebricher (2009), Obervation and analysis of Polar Mesospheric Winter Echoes modulated by artificial electron heating, paper presented at 19th ESA Symposium on European Rocket and Balloon Programmes and Related Research, Eur. Space Agency, Bad Reichenhall, Germany.

Hervig, M. E., L. L. Gordley, L. E. Deaver, D. E. Siskind, M. H. Stevens, J. M. Russell III, S. M. Bailey, L. Megner, and C. G. Bardeen (2009), First satellite observations of meteoric smoke in the middle atmosphere, Geophys. Res. Lett., 36, L18805, doi:10.1029/2009GL039737.

Hunten, D. M., R. P. Turco, and O. B. Toon (1980), Smoke and dust particles of meteoric origin in the mesosphere and stratosphere, J. Atmos. Sci., $37,1342-1357$.
Kalashnikova, O., M. Horanyi, G. E. Thomas, and O. B. Toon (2000), Meteoric smoke production in the atmosphere, Geophys. Res. Lett., 27, 3293-3296.

Kassa, M., O. Havnes., and E. Belova (2005), The effect of electron bite-outs on artificial electron heating and the PMSE overshoot, Ann. Geophys., 23, $1-11$.

Kavanagh, A. J., F. Honary, M. T. Rietveld, and A. Senior (2006), First observations of the artificial modulation of Polar Mesosphere Winter Echoes, Geophys. Res. Lett., 33, L19801, doi:10.1029/2006GL027565.

Kero, A., T. Bösinger, P. Pollari, E. Turunen, and M. Rietveld (2000), First EISCAT measurement of electron-gas temperature in the artificially heated D-region ionosphere, Ann. Geophys., 18, 1210-1215, doi:10.1007/s00585000-1210-8.

Kero, A., C. F. Enell, A. J. Kavanagh, J. Vierinen, I. Virtanen, and E. Turunen (2008), Could negative ion production explain the polar mesosphere winter echo (PMWE) modulation in active HF heating experiments?, Geophys. Res. Lett., 35, L23102, doi:10.1029/2008GL035798.

La Hoz, C., and O. Havnes (2008), Artificial modification of polar mesospheric winter echoes with an RF heater: Do charged dust particles play an active role?, J. Geophys. Res., 113, D19205, doi:10.1029/2008JD010460.

Lean, J. (1987), Irradiance variations: A review, J. Geophys. Res, 92(D1), 839-868.

Lübken, F.-J., B. Strelnikov, M. Rapp, W. Singer, R. Latteck, A. Brattli, U.-P. Hoppe, and M. Friedrich (2006), The thermal and dynamical state of the atmosphere during polar mesosphere winter echoes, Atmos. Chem. Phys., 6, 13-24.

Megner, L., M. Rapp, and J. Gumbel (2006), Distribution of meteoric smoke-Sensitive to microphysical properties and atmospheric conditions, Atmos. Chem. Phys., 6, 4415-4426.

Portnyagin, Y., et al. (2004) Mesosphere/lower thermosphere prevailing wind model, Adv. Space. Res., 34, 1755-1762.

Rapp, M. (2009), Charging of mesospheric aerosol particles: The role of photodetachment and photoionization from meteoric smoke and ice particles, Ann. Geophys., 27, 2417-2422.

Rapp, M., and F.-J. Lübken (2004), Polar mesosphere summer echoes (PMSE): Review of observations and current understanding, Atmos. Chem. Phys., 4, 2601-2633.

Rapp, M., et al. (2010), Rocket-borne in situ measurements of meteor smoke: Charging properties and implications for seasonal variation, J. Geophys. Res., 115, D00I16, doi:10.1029/2009JD012725.

Rietveld, M. T., H. K. Kohl, and H. Kopka (1993), Introduction to ionospheric heating at Tromsø. Part I: Experimental overview, J. Atmos. Terr. Phys., 55, 577-599.

Robertson, S., et al. (2009), Mass analysis of charged aerosol particles in NLC and PMSE during the ECOMA/MASS campaign, Ann. Geophys., 27, 1213-1232, doi:10.5194/angeo-27-1213-2009.

Rosinski, J., and R. H. Snow (1961), Secondary particulate matter from meteor vapors, J. Meteorol., 18, 736-745.

Routledge, G., M. J. Kosch, A. Senior, A. J. Kavanagh, I. W. McCrea, and M. T. Rietveld (2011), A statistical survey of electron temperature enhancements in heater modulated polar mesospheric summer echoes at EISCAT, J. Atmos. Terr. Phys., 73, 472-482, doi:10.1016/j.jastp.2010. 11.004

Scales, W. A., and C. Chen (2008), On initial enhancement of mesospheric dust associated plasma irregularities subsequent to radiowave heating, Ann. Geophys., 26, 2265-2271, doi:10.5194/angeo-26-2265-2008.

Shukla, P. D., and A. A. Mamun (2002), Introduction to Dusty Plasma Physics, Inst. of Phys., Bristol, U. K.

von Cossart, G., J. Fiedler, and U. von Zahn (1999), Size distributions of NLC particles as determined from 3-color observations of NLC by ground-based lidar, Geophys. Res. Lett., 26, 1513-1516.

Weingartner, J. C., and B. T. Draine (2001), Electron-ion recombination on grains and poycyclic aromatic hydrocarbons, Astrophys. J., 563, $842-852$.

Zeller, O., M. Zecha, J. Bremer, R. Latteck, and W. Singer (2006), Mean characteristics of mesospheric winter echoes at mid- and high latitudes, J. Atmos. Sol. Terr. Phys., 68, 1087-1104.

G. Baroni, A. Biebricher, O. Havnes, M. Kassa, and C. La Hoz, Institute of Physics and Technology, University of Tromsø, N-9037 Tromsø, Norway. (cesar.la.hoz@uit.no)

M. T. Rietveld, EISCAT Scientific Association, N-9027 Ramfjordbotn, Norway. 